\title{
Numerical Investigation of the Steady State of a Driven Thin Film Equation
}

\author{
A. J. Hutchinson, C. Harley, and E. Momoniat \\ Centre for Differential Equations, Continuum Mechanics and Applications, School of Computational and Applied \\ Mathematics, University of the Witwatersrand, Private Bag 3, Wits Johannesburg 2050, South Africa
}

Correspondence should be addressed to C. Harley; charis.harley@wits.ac.za

Received 6 November 2012; Accepted 29 December 2012

Academic Editor: Mehmet Pakdemirli

Copyright (C) 2013 A. J. Hutchinson et al. This is an open access article distributed under the Creative Commons Attribution License, which permits unrestricted use, distribution, and reproduction in any medium, provided the original work is properly cited.

\begin{abstract}
A third-order ordinary differential equation with application in the flow of a thin liquid film is considered. The boundary conditions come from Tanner's problem for the surface tension driven flow of a thin film. Symmetric and nonsymmetric finite difference schemes are implemented in order to obtain steady state solutions. We show that a central difference approximation to the third derivative in the model equation produces a solution curve with oscillations. A difference scheme based on a combination of forward and backward differences produces a smooth accurate solution curve. The stability of these schemes is analysed through the use of a von Neumann stability analysis.
\end{abstract}

\section{Introduction}

In this paper we investigate numerical solutions of the thirdorder ordinary differential equation (ODE)

$$
\frac{d^{3} y}{d x^{3}}=1-\frac{1}{y}+\frac{A}{y^{3}}
$$

where $A$ is a constant to be determined. Equation (1) is the steady state case of the partial differential equation

$$
u_{t}+f(u)_{x}=-\left(u^{3} u_{x x x}\right)_{x},
$$

where the flux is given by

$$
f(u)=u^{2}-u^{3} .
$$

This equation describes the flow of a thin liquid film, where $u(x, t) \geq 0$ denotes the film thickness. The flux terms represent surface shear and gravity, where the forces act in opposing directions, and the diffusion term on the right-hand side represents surface tension. The surface shear term may arise due to temperature or concentration gradients or to an external shear force. Equation (2) with (3) is a special case of the more general partial differential equation

$$
u_{t}+\left(u^{2}-\alpha u^{3}\right)_{x}=\beta\left(u^{3} u_{x}\right)_{x}-\gamma\left(u^{3} u_{x x x}\right)_{x}
$$

where $\alpha, \beta$, and $\gamma$ are nonnegative parameters. Equation (4) models the flow of a thin film of fluid with thickness $u(x, t)$ above an inclined plane. The variable $x$ is the distance down the plane, and the variable $t$ represents time. The parameters $\alpha, \beta$, and $\gamma$ describe the effects of surface tension, gravity, and the angle of inclination of the plane, respectively. This model is derived in the work by Bertozzi et al. [1], and numerical solutions are obtained and investigated. In our model, we consider a vertical plane, and thus $\beta=0$. With $\beta=0$ and by considering the steady state solution, that is, $u(x, t)=y(x)$, (4) reduces to

$$
\left(y^{2}-\alpha y^{3}\right)_{x}=-\gamma\left(y^{3} y_{x x x}\right)_{x} .
$$

Dividing (5) by $\gamma$ and, for simplicity, replacing $1 / \gamma$ and $\alpha / \gamma$ with $\alpha$ and $\beta$, respectively, (5) becomes

$$
\left(\alpha y^{2}-\beta y^{3}\right)_{x}=-\left(y^{3} y_{x x x}\right)_{x}
$$

which can be rewritten as follows:

$$
\frac{d}{d x}\left(y^{3} \frac{d^{3} y}{d x^{3}}+\alpha y^{2}-\beta y^{3}\right)=0 .
$$

In (7), the parameter $\alpha$ is proportional to Marangon's number which is a ratio of the surface tension to viscous forces, and 
the parameter $\beta$ is proportional to the Bond number which is a ratio of gravity to viscous forces [2]. The more general equation,

$$
\frac{d}{d x}\left(y^{k} \frac{d^{3} y}{d x^{3}}+\alpha y^{2}-\beta y^{3}\right)=0
$$

was solved numerically for the case where the arbitrary constant of integration was set equal to 1 , and the asymptotic solution was investigated in [2].

We can nondimensionalize the equation using the following transformations:

$$
y=\frac{\alpha}{\beta} \bar{y}, \quad x=\alpha^{(k-2) / 3} \beta^{(1-k) / 3} \bar{x}
$$

and after integrating once with respect to $x,(8)$ becomes

$$
y^{k} \frac{d^{3} y}{d x^{3}}+y^{2}-y^{3}=A
$$

The case where $A=1$ has been studied in [2]. We will consider (10) for the case $k=3$, where the value of $A$ will be determined through the application of one of the boundary conditions. In this paper, we use the conditions provided by Tanner's problem [3] in the spreading of silicon oil drops on a horizontal surface modelled as a thin film. These conditions are given by

$$
y(0)=1, \quad y^{\prime}(0)=0
$$

In his work Tanner [3] investigated surface tension-driven spreading of an oil drop modelled as a thin film. The problem is closed by the boundary condition

$$
y(r)=0
$$

where $r$ is a positive constant. The autonomous nature of (10) makes the specification of $r$ arbitrary. The boundary condition (12) describes the physical phenomenon of touchdown [4] or, more precisely, the point at which the free surface of the thin film makes contact with the solid substrate. Given that (1) is singular at $y=0$, the boundary condition (12) is imposed as

$$
y(r)=\epsilon,
$$

where $\epsilon \ll 1$ is the height of the precursor film. A discussion of this approximation can be found in Bertozzi $[5,6]$.

In this paper, we use a symmetric and nonsymmetric finite difference scheme to solve (1). This method was applied in [4] to the following equation:

$$
\frac{d^{3} y}{d x^{3}}=y^{-k}
$$

In [7] a shooting method was applied to (14), and the asymptotic solution was also studied. In this research, we will conduct a von Neumann stability analysis as a means of investigating the stability of the methods employed.

This paper is set out as follows: in Section 2.1 we implement a symmetric finite difference approximation to the third derivative with truncation error $O\left(h^{2}\right)$. We see that this scheme is zero stable and a spectral analysis gives inconclusive results. Thus, a von Neumann stability analysis is implemented. The solution obtained via the symmetric difference scheme contains oscillations. In Section 2.2 we implement a non-symmetric difference approximation to the third derivative. This scheme has truncation error $O(h)$. It is shown to be zero stable; however, a von Neumann stability analysis is also implemented. The solution of the non-symmetric difference scheme does not contain any oscillations.

We do not rigorously prove that the schemes investigated in this paper converge, but rather show that the schemes are stable. Furthermore, we show that convergence does take place by evaluating the absolute error at each iteration. We show, in a similar fashion to Momoniat [4], that even if a numerical scheme is stable, the boundary conditions produce results which are physically unstable. In this instance, this instability manifests as oscillations in the solution. Furthermore, stability and accuracy should not necessarily be accessed based upon the truncation error of the scheme: in this case the most accurate and stable scheme is the nonsymmetric scheme with a truncation error of $O(h)$ and a stability criteria of $h<0.03$, instead of $O\left(h^{2}\right)$ and $h<0.02$ as for the symmetric scheme where the solution curve exhibited oscillations.

\section{Finite Difference Methods}

In the sections that follow, we let $A=\epsilon^{2}-\epsilon^{3}$ and $\epsilon=10^{-3}$. The choice of $A$ comes from a consideration of the equation at $x=r$, where

$$
\left.y^{k} \frac{d^{3} y}{d x^{3}}\right|_{x=r}=y^{3}-y^{2}+\left.A\right|_{x=r} .
$$

We may claim; however, that $d^{3} y /\left.d x^{3}\right|_{x=r}=0$ which means that $A=-\left.\left(y^{3}-y^{2}\right)\right|_{x=r}=-\left(\epsilon^{3}-\epsilon^{2}\right)$ since $y(r)=\epsilon$. Doing so makes sense given the implementation of the physical condition that the volume flux at $y=\epsilon$ vanishes, where $\epsilon$ is the height of the precursor film. Thus, we may choose $A$ such that (10) becomes

$$
y^{k} \frac{d^{3} y}{d x^{3}}=y^{3}-y^{2}-\left(\epsilon^{3}-\epsilon^{2}\right) .
$$

2.1. Symmetric Difference Approximation. We approximate (1) subject to the boundary conditions given by (11) and (13) by implementing a symmetric difference approximation. We define $y_{i}=y\left(x_{i}\right)$ to be the approximate value of $y$ at a point $x_{i}$, where $x_{i}=i h, i=0,1, \ldots, n, x_{0}=0$, and $x_{n}=r$, and the step length $h$ is given by $h=r / n$, where $n$ is a positive integer. The first derivative $y^{\prime}$ at a point $x_{i}$ is approximated as

$$
y_{i}^{\prime}=\frac{y_{i+1}-y_{i-1}}{2 h}+O\left(h^{2}\right),
$$

and the second derivative $y^{\prime \prime}$ at a point $x_{i}$ is approximated as

$$
y_{i}^{\prime \prime}=\frac{y_{i+1}-2 y_{i}+y_{i-1}}{h^{2}}+O\left(h^{2}\right) .
$$


The third derivative $y^{\prime \prime \prime}$ is approximated by a central difference approximation,

$$
y_{i}^{\prime \prime \prime}=\frac{y_{i+1}^{\prime \prime}-y_{i-1}^{\prime \prime}}{2 h}+O\left(h^{2}\right),
$$

and using (18) we obtain

$$
y_{i}^{\prime \prime \prime}=\frac{y_{i+2}-2 y_{i+1}+y_{i}-y_{i}+2 y_{i-1}-y_{i-2}}{2 h^{3}}+O\left(h^{2}\right) .
$$

Simplifying this we get

$$
y_{i}^{\prime \prime \prime}=\frac{y_{i+2}-2 y_{i+1}+2 y_{i-1}-y_{i-2}}{2 h^{3}}+O\left(h^{2}\right) .
$$

Approximating (1) using (21) and after simplifying, we obtain a nonlinear difference equation,

$$
y_{i+2}-2 y_{i+1}+2 y_{i-1}-y_{i-2}=2 h^{3}\left(1-\frac{1}{y_{i}}+\frac{A}{y_{i}^{3}}\right),
$$

with a truncation error of $O\left(h^{2}\right)$. We now define $y_{i}^{(j)}$ to be the $j$ th iteration value to $y_{i}$. Using this notation, $(22)$ can be evaluated iteratively in the following manner:

$$
\begin{aligned}
y_{i+2}^{(j+1)} & -2 y_{i+1}^{(j+1)}+2 y_{i-1}^{(j+1)}-2 y_{i-2}^{(j+1)} \\
& =2 h^{3}\left(1-\frac{1}{y_{i}^{(j)}}+\frac{A}{y_{i}^{(j)^{3}}}\right) .
\end{aligned}
$$

The boundary conditions are incorporated by requiring that $y_{i}^{(0)}$ satisfies (11) and (13). The boundary conditions $y(0)=1$ and $y(r)=\epsilon$ are implemented by

$$
y_{0}=1, \quad y_{n}=\epsilon
$$

When evaluating (23) at the point $x_{n}=(n-1) h$, a value for $y_{n+1}$ is required. In thin film theory, it is reasonable to suppose that the height $y$ of the fluid at position $x_{n}$ is the same at $x_{n+1}$ [4]. In other words, we can obtain a value for $y_{n+1}$ by setting

$$
y_{n+1}=y_{n}=\epsilon .
$$

Also, in order to calculate $y_{1}$ using (25), a value for $y_{-1}$ is required. A central difference approximation is used to approximate the boundary condition $y^{\prime}(0)=0$,

$$
y_{0}^{\prime}=\frac{y_{1}-y_{-1}}{2 h}
$$

and setting (26) equal to zero, we have

$$
y_{-1}=y_{1}
$$

The difference equation (23), together with the boundary conditions (24), (25), and (27), can be written more compactly in matrix form as follows:

$$
A \underline{y}^{(j+1)}=\underline{b}
$$

where

$$
\begin{aligned}
& A=\left[\begin{array}{ccccccccccccc}
1 & 0 & 0 & 0 & 0 & 0 & \ldots & 0 & 0 & 0 & 0 & 0 & 0 \\
2 & -1 & -2 & 1 & 0 & 0 & \ldots & 0 & 0 & 0 & 0 & 0 & 0 \\
-1 & 2 & 0 & -2 & 1 & 0 & \ldots & 0 & 0 & 0 & 0 & 0 & 0 \\
0 & -1 & 2 & 0 & -2 & 0 & \ldots & 0 & 0 & 0 & 0 & 0 & 0 \\
\vdots & \vdots & \vdots & \vdots & \vdots & \vdots & \ldots & \vdots & \vdots & \vdots & \vdots & \vdots & \vdots \\
0 & 0 & 0 & 0 & 0 & 0 & \ldots & -1 & 2 & 0 & -2 & 1 & 0 \\
0 & 0 & 0 & 0 & 0 & 0 & \ldots & 0 & -1 & 2 & 0 & -2 & 1 \\
0 & 0 & 0 & 0 & 0 & 0 & \ldots & 0 & 0 & -1 & 2 & 0 & -1 \\
0 & 0 & 0 & 0 & 0 & 0 & \ldots & 0 & 0 & 0 & 0 & 0 & 1
\end{array}\right], \\
& \underline{y}^{(j+1)}=\left[y_{0}^{(j+1)}, y_{1}^{(j+1)}, y_{2}^{(j+1)}, y_{3}^{(j+1)}, \ldots, y_{n-1}^{(j+1)}, y_{n}^{(j+1)}\right]^{T} \text {, } \\
& \underline{b}=\left[1, h^{3}\left(1-\frac{1}{y_{1}^{(j)}}+\frac{A}{y_{1}^{(j) 3}}\right), h^{3}\left(1-\frac{1}{y_{2}^{(j)}}+\frac{A}{y_{2}^{(j) 3}}\right), \ldots,\right. \\
& \left.h^{3}\left(1-\frac{1}{y_{n-1}^{(j)}}+\frac{A}{y_{n-1}^{(j) 3}}\right), \epsilon\right]^{T} \text {. }
\end{aligned}
$$

An initial guess, $y_{i}^{(0)}$, to $y$ that satisfies the boundary conditions (11) and (13) is given by

$$
y_{i}^{(0)}=1+(\epsilon-1) x_{i}^{2} .
$$

The corresponding homogeneous equation of (23) is given by

$$
y_{i+2}-2 y_{i+1}+2 y_{i-1}-y_{i-2}=0 \text {. }
$$

This equation has been studied extensively in [4]. This difference scheme is zero stable, and the results of a spectral analysis were found to be inconclusive with regard to the convergence of the difference scheme. Plots of (31) were found to contain oscillations.

Given that we need to consider an alternative form of analysis we choose to implement the von Neumann stability analysis. In order to do so we first need to linearize our scheme (23). We notice that the difference scheme is linear in $y$ except for the $\left(1-1 / y_{i}^{(j)}+A / y_{i}^{(j)^{3}}\right)$ term. Rewriting (23) we obtain

$$
\begin{aligned}
y_{i+2}^{(j+1)} & -2 y_{i+1}^{(j+1)}+2 y_{i-1}^{(j+1)}+y_{i-2}^{(j+1)} \\
& =2 h^{3} y_{i}^{j}\left(\frac{1}{y_{i}^{(j)}}-\frac{1}{y_{i}^{(j)^{2}}}+\frac{A}{y_{i}^{(j)^{4}}}\right) .
\end{aligned}
$$

Let

$$
\gamma=2 h^{3}\left(\frac{1}{y_{i}^{(j)}}-\frac{1}{y_{i}^{(j)^{2}}}+\frac{A}{y_{i}^{(j)^{4}}}\right),
$$

and by making the substitution (33) in our difference scheme (32) we obtain

$$
y_{i+2}^{(j+1)}-2 y_{i+1}^{(j+1)}+2 y_{i-1}^{(j+1)}+y_{i-2}^{(j+1)}=\gamma y_{i}^{(j)},
$$

which is now linear in $y$. 
We now implement a Von Neumann stability analysis by making the substitution,

$$
y_{i}^{(j)}=Y^{j} e^{I \omega i h},
$$

into (34), where $I^{2}=-1$ and $\omega$ is a constant. The simplification that follows here can be found in [4], where $\gamma$ is given by

$$
2 h^{3} y_{i}^{(j)^{-k-1}}
$$

whereas, in this paper, $\gamma$ is given by (33). In [4] $\gamma>0$; however, the sign of $\gamma$ defined by (33) is not as straightforward to determine. Following the same procedure as described in [4] we obtain the following expression for the amplification factor:

$$
|g|=\frac{|\gamma|}{\left|8 \sin ^{2}(\omega h / 2) \sin (\omega h)\right|} .
$$

This can be further simplified, resulting in an upper bound for $\gamma$ given by

$$
|\gamma|<3 \sqrt{3}
$$

In order to obtain the maximum and minimum values of $\gamma$, consider the plots of $|\gamma|$ as a function of $y$ given in Figures 1 and 2 . We see that the minimum value for $|\gamma|$ is obtained at $y=\epsilon$ and is zero. In order to obtain the value at which $|\gamma|$ is a maximum, we find the second derivative of $\gamma(y)$ and set it equal to zero. Solving this in Mathematica, we find that $|\gamma|$ attains its maximum value at $y=0.001414$. The value of $\left|\gamma\left(y_{m}\right)\right|$ is 249543. In other words, we have deduced the following results:

$$
\gamma_{\max }=\gamma\left(y_{\max }\right) \approx 499086 h^{3}, \quad \gamma_{\min }=0 .
$$

Now $\left|\gamma_{\min }\right|$ always satisfies (38). Set $\gamma=\gamma_{\max }$ to obtain

$$
h<\left[\frac{3 \sqrt{3}}{499086}\right]^{1 / 3} \approx 0.02 .
$$

The von Neumann stability criteria is satisfied if (40) is satisfied. We can, therefore, conclude that the iteration (32) is conditionally stable, provided the value of the step length remains below the maximum value of 0.02 . In Table 1 we show the absolute error at each iteration. After five iterations the absolute error max $\left|y^{(j+1)}-y^{(j)}\right|$ is $O\left(10^{-9}\right)$. We plot the results after five iterations in Figure 3. In both Table 1 and Figure 3 we have chosen $h=1 / n$, where $n=100$, and thus, the choice of $h=10^{-2}$ satisfies the von Neumann stability criteria.

The solution obtained via a symmetric difference scheme given by (23) oscillates. These oscillations diminish as the value of the step length $h$ is reduced. However, even for $n=$ 1000 there are still oscillations present. This seems to indicate that the oscillations occur in the homogeneous part of the difference scheme. Similar to [4] we believe these oscillations occur as a result of imposing the additional boundary condition necessary to iterate the numerical scheme. In the next

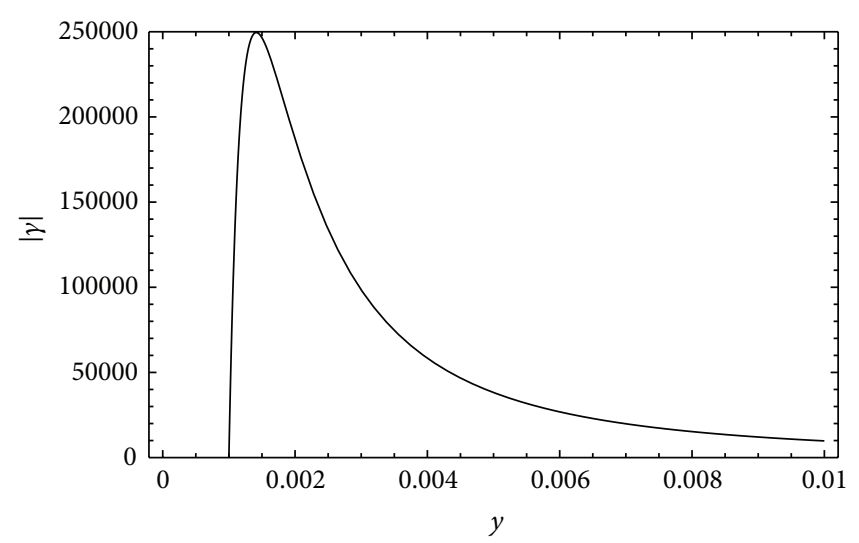

Figure 1: Plot of $|\gamma(y)|$ for $\epsilon \leq y \leq 0.01$.

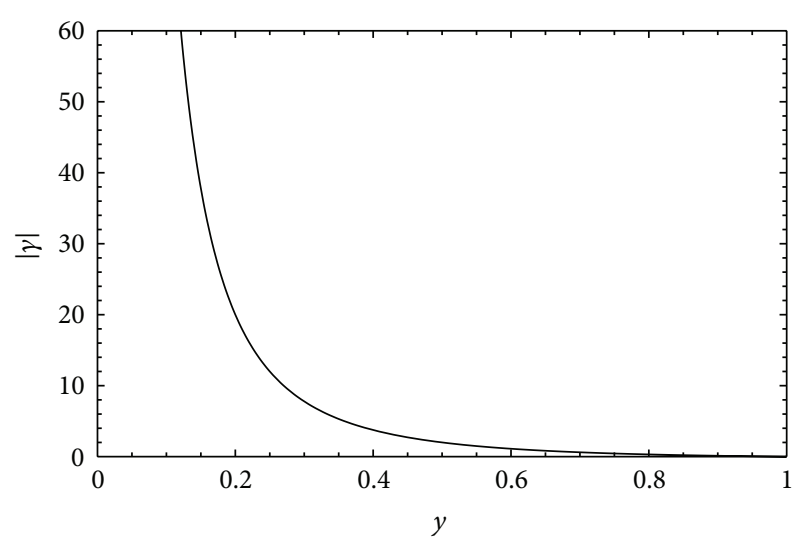

Figure 2: Plot of $|\gamma(y)|$ for $\epsilon \leq y \leq 1$.

TABLE 1: Table showing the absolute error after each iteration of (28), where $n=100$.

\begin{tabular}{lc}
\hline$i$ & $\begin{array}{c}\text { Error } \\
\max \left|\underline{y}^{(j+1)}-\underline{y}^{(j)}\right|\end{array}$ \\
\hline 1 & $2.4879 e-02$ \\
2 & $3.9646 e-04$ \\
3 & $8.5598 e-06$ \\
4 & $2.3696 e-07$ \\
5 & $7.4744 e-09$ \\
\hline
\end{tabular}

section we show that a non-symmetric difference approximation provides smooth accurate solutions even though the truncation error is higher than the truncation error for the symmetric difference scheme.

2.2. Nonsymmetric Difference Approximation. An alternative method to approximate the third-order ODE (1) is considered below. Forward and backward difference approximations are used to approximate the first- and second-order derivatives 


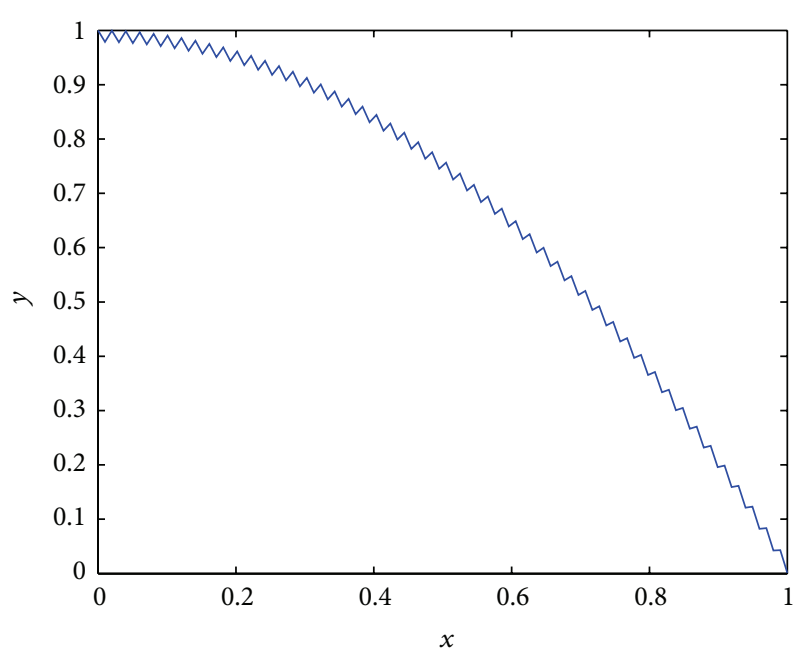

Figure 3: Solution to the symmetric difference scheme for $n=100$.

TABLE 2: Table showing the absolute error after each iteration of (50), where $n=100$.

\begin{tabular}{lc}
\hline$i$ & $\begin{array}{c}\text { Error } \\
\max \left|\underline{y}^{(j+1)}-\underline{y}^{(j)}\right|\end{array}$ \\
\hline 1 & $1.0483 e-02$ \\
2 & $4.1902 e-04$ \\
3 & $1.6225 e-05$ \\
4 & $6.2815 e-07$ \\
5 & $2.4313 e-08$ \\
6 & $9.4100 e-10$ \\
\hline
\end{tabular}

instead of the central (symmetric) approximations considered above. The approximations are given by

$$
\begin{aligned}
y_{i}^{\prime}= & \frac{y_{i+1}-y_{i}}{h}+O(h), \\
y_{i}^{\prime \prime}= & \frac{\left(y_{i+1}-y_{i}\right) / h-\left(y_{i}-y_{i-1}\right) / h}{h}+O\left(h^{2}\right), \\
y_{i}^{\prime \prime}= & \frac{y_{i+1}-2 y_{i}+y_{i-1}}{h^{2}}+O\left(h^{2}\right), \\
y_{i}^{\prime \prime \prime}= & \frac{\left(y_{i+1}-y_{i}\right) / h-2\left(\left(y_{i}-y_{i-1}\right) / h\right)+\left(y_{i-1}-y_{i-2}\right) / h}{h^{2}} \\
& +O(h), \\
y_{i}^{\prime \prime \prime}= & \frac{y_{i+1}-3 y_{i}+3 y_{i-1}-y_{i-2}}{h^{3}}+O(h) .
\end{aligned}
$$

Substituting (45) into (1) results in the following difference equation:

$$
\frac{y_{i+1}-3 y_{i}+3 y_{i-1}-y_{i-2}}{h^{3}}=\left(1-\frac{1}{y_{i}}+\frac{A}{y_{i}^{3}}\right),
$$

which has a truncation error of $O(h)$. After multiplying by $h^{3}$ we obtain

$$
y_{i+1}-3 y_{i}+3 y_{i-1}-y_{i-2}=h^{3}\left(1-\frac{1}{y_{i}}+\frac{A}{y_{i}^{3}}\right) .
$$

Equation (47) can be evaluated iteratively as

$$
y_{i+1}^{(j+1)}-3 y_{i}^{(j+1)}+3 y_{i-1}^{(j+1)}-y_{i-2}^{(j+1)}=h^{3}\left(1-\frac{1}{y_{i}^{(j)}}+\frac{A}{y_{i}^{(j) 3}}\right) \text {. }
$$

To check for 0 stability, we consider the following equation:

$$
y_{i+1}-3 y_{i}+3 y_{i-1}-y_{i-2}=0,
$$

which is the homogeneous part of (47). This equation has been studied extensively in [4]. This scheme was proven to be zero stable, and no oscillations were present in the solution curve. We can write the above system in the form

$$
B \underline{y}^{(j+1)}=\underline{b},
$$

where

$$
\begin{gathered}
\quad=\left[\begin{array}{cccccccccccc}
1 & 0 & 0 & 0 & 0 & \ldots & 0 & 0 & 0 & 0 & 0 & 0 \\
3 & -4 & 1 & 0 & 0 & \ldots & 0 & 0 & 0 & 0 & 0 & 0 \\
-1 & 3 & -3 & 1 & 0 & \ldots & 0 & 0 & 0 & 0 & 0 & 0 \\
0 & -1 & 3 & -3 & 1 & \ldots & 0 & 0 & 0 & 0 & 0 & 0 \\
\vdots & \vdots & \vdots & \vdots & \vdots & \ldots & \vdots & \vdots & \vdots & \vdots & \vdots & \vdots \\
0 & 0 & 0 & 0 & 0 & \ldots & -1 & 3 & -3 & 1 & 0 & 0 \\
0 & 0 & 0 & 0 & 0 & \ldots & 0 & -1 & 3 & -3 & 1 & 0 \\
0 & 0 & 0 & 0 & 0 & \ldots & 0 & 0 & -1 & 3 & -3 & 1 \\
0 & 0 & 0 & 0 & 0 & \ldots & 0 & 0 & 0 & 0 & 0 & 1
\end{array}\right], \\
\underline{y}^{(j+1)}=\left[y_{0}^{(j+1)}, y_{1}^{(j+1)}, y_{2}^{(j+1)}, y_{3}^{(j+1)}, \ldots, y_{n-1}^{(j+1)}, y_{n}^{(j+1)}\right]^{T}, \\
\underline{b}=\left[1, h^{3}\left(1-\frac{1}{y_{1}^{(j)}}+\frac{A}{y_{1}^{(j) 3}}\right), h^{3}\left(1-\frac{1}{y_{2}^{(j)}}+\frac{A}{y_{2}^{(j) 3}}\right), \ldots,\right. \\
\left.h^{3}\left(1-\frac{1}{y_{n-1}^{(j)}}+\frac{A}{y_{n-1}^{(j) 3}}\right), \epsilon\right]^{T} .
\end{gathered}
$$

An initial guess satisfying the boundary conditions (11) and (13) (as before) is

$$
y_{i}^{(0)}=1+(\epsilon-1) x_{i}^{2} .
$$

A von Neumann stability analysis was used to determine the stability of the system (48). To perform the analysis equation (48) was linearized as follows:

$$
y_{i+1}^{(j+1)}-3 y_{i}^{(j+1)}+3 y_{i-1}^{(j+1)}-y_{i-2}^{(j+1)}=\beta y_{i}^{(j)},
$$

where

$$
\beta=h^{3}\left(\frac{1}{y_{i}^{(j)}}-\frac{1}{y_{i}^{(j)^{2}}}+\frac{A}{y_{i}^{(j)^{4}}}\right) .
$$




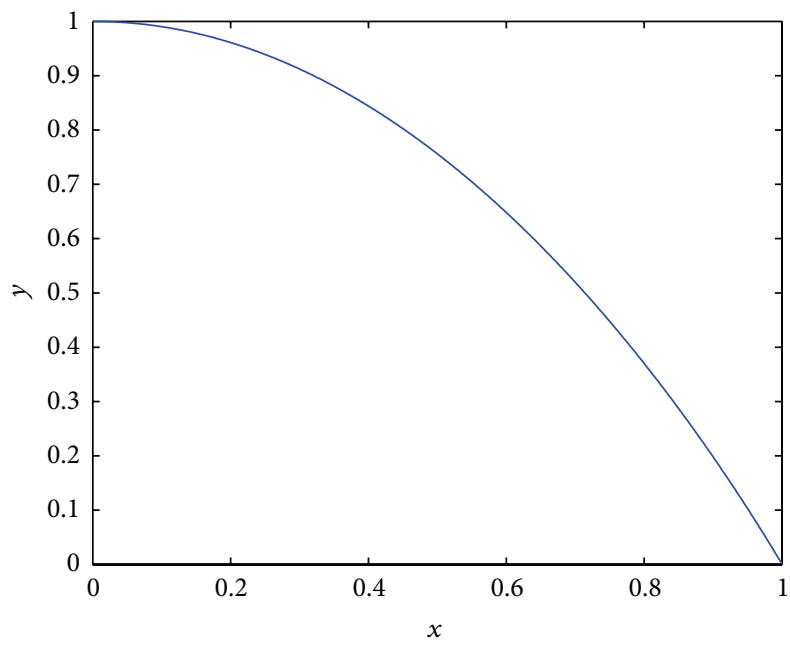

Figure 4: Solution to the nonsymmetric scheme for $n=100$.

In [4] the value assigned to $\beta$ is $h^{3} y_{i}^{(j)^{-k-1}}$. The analysis follows the same procedure with the value for $\beta$ defined by (54) replacing the value for $\beta$ in [4]. The amplification factor, $g$, is given by

$$
g=\frac{|\beta|}{8 \sin ^{3}(\omega h / 2)} .
$$

An upper bound for $g$ is given by

$$
g<8 \beta \text {. }
$$

Using the method for determining the maximum and minimum values for $\gamma$ from the previous section, we have that a similar computation for $\beta$ yields

$$
\beta_{\min }=0, \quad \beta_{\max }=249543 h^{3} .
$$

The von Neumann stability criteria give that

$$
h<\left(\frac{3 \sqrt{3}}{249543}\right)^{1 / 3} \approx 0.03
$$

which shows that the scheme is conditionally stable. In Table 2 we show the absolute error $\max \left|y^{(j+1)}-y^{(j)}\right|$ at each iteration for six iterations-after six iterations the absolute error is $O\left(10^{-10}\right)$. As done previously, in both Table 2 and Figure 3 we have chosen $h=1 / n$, where $n=100$ such that the choice of $h=10^{-2}$ satisfies the von Neumann stability criteria. A plot of the nonsymmetric difference scheme is given in Figure 4 where it can be seen that there are no oscillations present.

\section{Concluding Remarks}

In order to investigate the steady state solutions of the partial differential equation (2) we integrate with respect to $x$. In doing so an arbitrary constant arises, denoted by $A$, the value of which was deduced by using the physical condition that the volume flux at $y=\epsilon$ vanishes. Symmetric and nonsymmetric finite difference schemes were implemented in order to obtain steady state solutions. In this paper, we use 0 stability to show that the finite difference schemes used to approximate the third-order derivative in the model equation are stable. A von Neumann stability analysis is implemented to obtain criteria on the step length which makes the iterative evaluation of the nonlinear difference scheme stable.

The symmetric difference scheme has a truncation error $O\left(h^{2}\right)$ and is shown to be 0 -stable. The nonlinear difference scheme is solved iteratively and displays unstable results even though the step length maintains the von Neumann stability criteria. These oscillations persist even as $h$ is reduced. The non-symmetric difference scheme, which has a truncation error of $O(h)$ and is 0 stable, in turn provided a stable solution. The results obtained in this paper reiterate the fact that finite difference approximations with a small truncation error should not thoughtlessly be used to solve problems in which the boundary conditions affect the behaviour of the solution.

\section{Acknowledgment}

C. Harley acknowledges support from the National Research Foundation, South Africa, under Grant no. 79184.

\section{References}

[1] A. L. Bertozzi, A. Münch, and M. Shearer, "Undercompressive shocks in thin film flows," Physica D, vol. 134, no. 4, pp. 431-464, 1999.

[2] E. Momoniat, "A nonlinear eigenvalue problem from thin-film flow," Journal of Engineering Mathematics, 2012.

[3] L. H. Tanner, "The spreading of silicone oil drops on horizontal surfaces," Journal of Physics D, vol. 12, pp. 1473-1484, 1979.

[4] E. Momoniat, "Numerical investigation of a third-order ODE from thin film flow," Meccanica, vol. 46, no. 2, pp. 313-323, 2011.

[5] A. L. Bertozzi, "Symmetric singularity formation in lubricationtype equations for interface motion," SIAM Journal on Applied Mathematics, vol. 56, no. 3, pp. 681-714, 1996.

[6] A. L. Bertozzi, "The mathematics of moving contact lines in thin liquid films," Notices of the American Mathematical Society, vol. 45, no. 6, pp. 689-697, 1998.

[7] E. Momoniat, "On the determination of the steady film profile for a non-Newtonian thin droplet," Computers \& Mathematics with Applications, vol. 62, no. 1, pp. 383-391, 2011. 


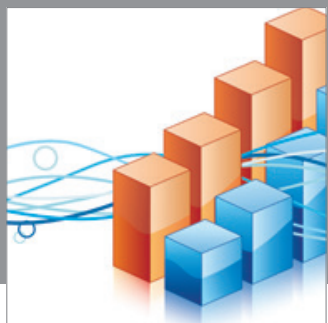

Advances in

Operations Research

mansans

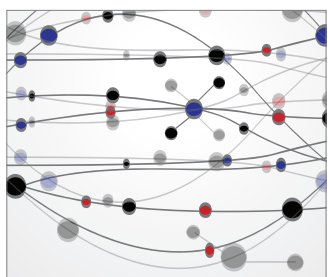

The Scientific World Journal
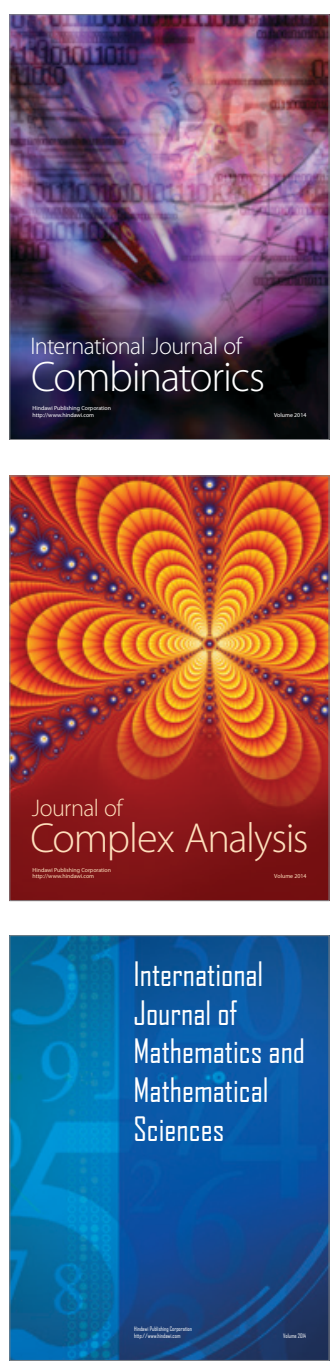
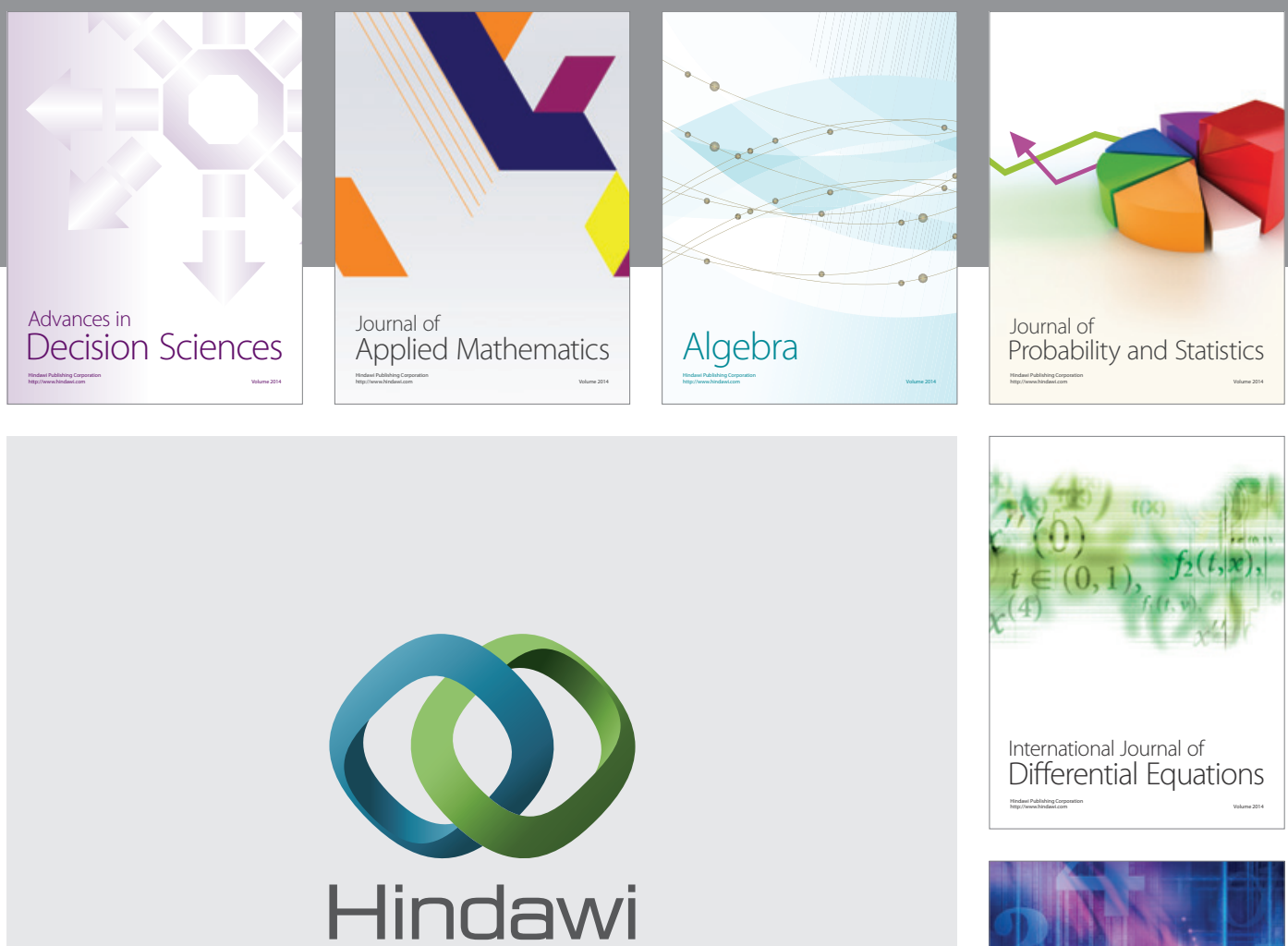

Submit your manuscripts at http://www.hindawi.com
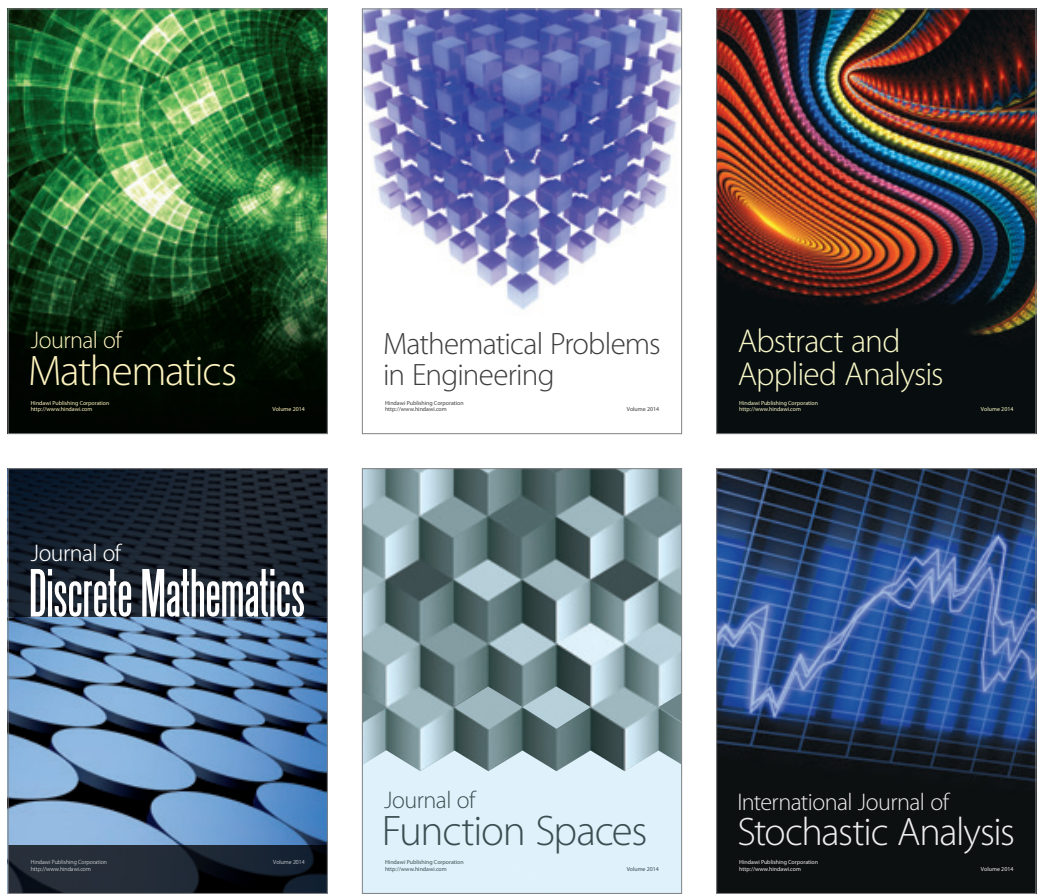

Journal of

Function Spaces

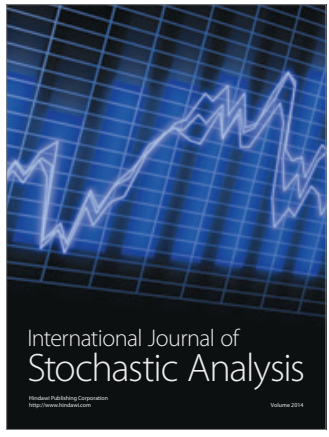

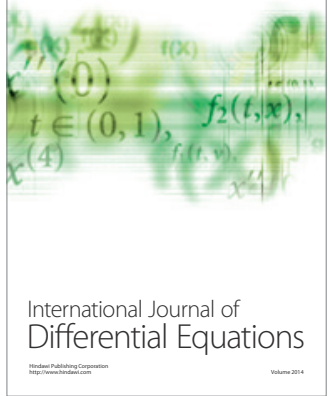
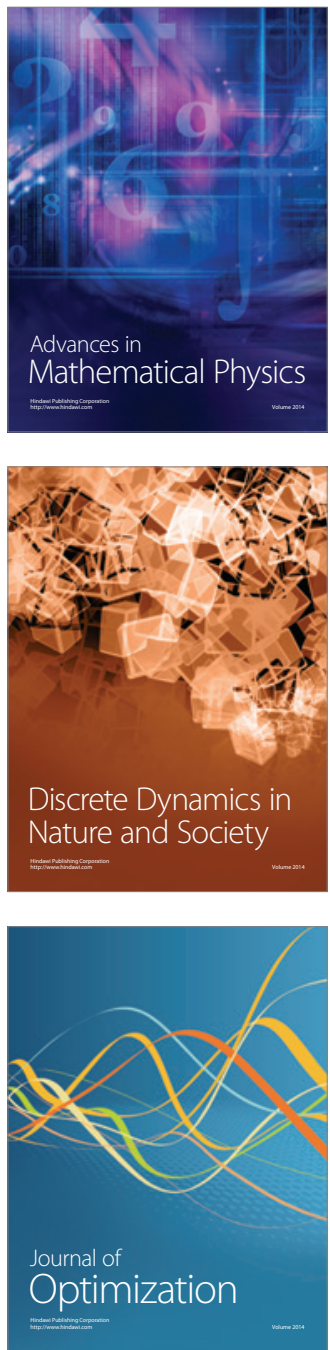